\title{
SENSOR PLACEMENT STRATEGIES FOR CONTAMINATION IDENTIFICATION IN WATER DISTRIBUTION NETWORKS: A REVIEW
}

\author{
OLUWASEYE SAMSON ADEDOJA ${ }^{1,2}$, YSKANDAR HAMAM ${ }^{1,2,3}$, BASET KHALAF ${ }^{1,2}$ \& ROTIMI SADIKU \\ ${ }^{1}$ Department Electrical Engineering, Tshwane University of Technology, South Africa \\ ${ }^{2}$ French South African Institute of Technology (F'SATI), Tshwane University of Technology, South Africa \\ ${ }^{3}$ Ecole Supérieure d'Ingénieurs en Électrotechnique et Électronique, France \\ ${ }^{4}$ Department of Chemical, Metallurgy and Material Engineering, Tshwane University of Technology, South Africa
}

\begin{abstract}
Safeguarding of water distribution networks is gaining attention due to the socio-economic implication of consuming contaminated water. An installation of water quality sensors has been recognised as one of the measures to minimise the distress. Notably, the procurement and maintenance cost of the water quality sensors have restrained the number of sensors to deploy across the network. This constraint means that the sensor placement strategy has to receive significant consideration. Over the years, researchers have proposed several techniques to handle the challenge. Each of the techniques has its shortcomings which must be addressed. This study presents a critical review of the sensor placement strategies in a water distribution network. The review results expressed the technical challenges, and proposed feasible solutions. The future research directions are also provided.

Keywords: sensor placement, water distribution network, water quality sensor, strategies, contamination, identification.
\end{abstract}

\section{INTRODUCTION}

The aim of a utility operator is to ensure potable water is distributed to consumers in adequate quantity and quality when desired. Excitingly, this also conforms with one of the major goals (target 6a) of the United Nation's sustainable Development Goal (SDG) in order to make world a better place for all, by 2030. Despite reports that access to water is gradually improving, a depleted delivery of potable water is a significant concern affecting most of the continents. Usually, the quality of water is examined at the treatment plant before being distributed to various consumer points. Nevertheless, its quality may considerably be contaminated during transportation from the treatment plant to the consumers' taps through cross-connections and pipe leakages. The complex nature of Water Distribution Networks (WDNs) allow human interference and therefore, vulnerable to accidental and intentional attacks [1]. Numerous reports have evidently shown that the socio-economic implications of such attacks can be disastrous [2]-[4]. In recent times, contamination incident in WDNs has been debated as one of the most unhealthy menace to the society that must be addressed [1]-[4].

Effective protection of WDNs is of paramount importance and its continuous surveillance is non-negotiable. One promising approach to monitor and safeguard the network is the deployment of water quality sensors across the network. If all nodes in the network can be thoroughly monitored, then a modest degree of protection can be assured [5]. From practical point of view, it is impossible to install sensors at every nodes because of the high procurement cost and budget constraint. Hence, the significant attention the sensor placement strategies has received. Similar techniques are applicable to pressure control valves and leakage detection in WDNs which have been broadly reported by several authors [6]-[10]. 
Basically, the research activities of the sensor placement strategies are designed to perform two major purposes. These are: (1) to minimise the number of sensors to deploy on the network and (2) to curtail the related effect of consuming contaminated water. Obviously, a quick identification of contaminant by the water quality sensors will enormously provide control strategies that will prevent the public from further consuming contaminated water. Therefore, it is crucial to conduct a critical review of this interesting topic. Some researchers [11]-[13] have previously presented survey in this field. However, an up till date investigation is inevitable. In this study, a critical review of the sensor placement strategies in WDN is presented. The contributions of this study include: (1) to outline the existing approaches; (2) to summarise the associated technical challenges; (3) to propose probable solutions; and (4) to enlist areas of future research direction. The remainder of the paper is as follow: Section 2 exhibits the classification of sensor placement objectives. In Section 3, existing solution techniques are outlined while Section 4 presents the technical challenges and probable solutions. Future research direction are highlighted in Section 5, while conclusions are provided in Section 6.

\section{CLASSIFICATION OF SENSOR PLACEMENT OBJECTIVES}

Continuous monitoring of WDNs is sacrosanct. However, various competitive performance objectives are considered in sensor placement strategies. Preferably, minimising the effect on the public health is one of the generally accepted objectives. Over the years, water quality sensors are utilised to monitor the WDNs and various performance objectives are evaluated. These objectives are related to: (i) quick identification of contamination occurrence, i.e. time to detection (TD); (ii) minimising the effect of contamination occurrence, for instance: volume of water consumed (VC), the population exposed to contamination (PE), and the extent of the contamination (EC). Broadly speaking, contaminant can intrude into the WDN at any particular location, and this necessitate that all nodes should be under surveillance. Unfortunately, this is mostly infeasible due to the limited budget constraints. Additional efforts were made to prevent the society under the limited available resources by considering the following: (i) related threat and/or (ii) maximising detection likelihood (DL) occurrence. There is a probability that sensor can raise false alarm if the contaminant concentration is below permissible limit. The delay in response time is also possible to occur. This can lead to an additional consideration of other performance objectives, such as: minimisation of sensor response time (SRT), minimisation of number of failed detections (NFD)/minimisation of probability of failed detection (PFD) and sensor detection redundancy (SDR). The strategies of sensor placement are broadly classified into two classes as single objective and multiobjective.

\subsection{Single placement objective}

A quick detection of contamination incident plays a significant role in minimising the effect on the society. This is generally referred to as "Time to Detection" (TD). The study by Kumar et al. [14] defined TD as the elapsed time between the start of the contaminant and its detection. The work proposed by Rathi and Gupta [15] discussed viability to minimise the time-detection. The authors suggested that the Level of Service (LOS) is directly proportional to the number of monitoring stations. A water distribution network from Nagpur City, India was employed as a case study. Hu et al. [16] developed a co-evolutionary optimisation approach to minimise an average time-detection of the contamination occurrence in a water distribution network. The effectiveness of the approach was demonstrated on a standard water 
network and the results verified the efficacy of the approach. TD is an important objective that can not be left out even in the multi-objective formulations.

Similarly, the quality of water can be assumed secured if large percentage of emanated supply nodes are protected. The study by Lee and Deininger [17] defined the Demand Coverage (DC) as the total demand of all those nodes, which can be presumed to be secured once the quality of water at the monitoring node is certified. Lee and Deininger [17] and Liu et al. [18] have employed an integer programming method and generic algorithm by incorporating EPNAET to address the problem. Also, Kumar et al. [19] proposed a mixed integer programming strategy to sensor placement challenge with focus to maximise the demand coverage under an assumed steady condition of the network. Thereafter, Propato et al. [20] and Berry et al. [21] improved on the demand coverage principles that involve the water quality and the time dependence in the demands.

Minimising the impact of contamination is also a major concern that must be considered. Kessler et al. [22] defined the total volume of contaminated water consumed (VC) ahead detection in order to evaluate the effect of the contamination. The authors advocated for the use of a pre-specified value of VC as a LOS. They came up with a pollution matrix for a specific LOS and placed an optimal number of monitoring stations that accommodated different contamination scenarios. A random multiple approach was considered by Ostfeld and Salomons [23] in order to determine location of the monitoring stations. An overview of the single objective in sensor placement strategies is depicted in Table 1.

Table 1: Overview of the single performance objective adapted from [12].

\begin{tabular}{llll}
\hline Objective & Approach & WQS & Reference \\
\hline TD & Heuristic & DHS & {$[14]$} \\
& Heuristic & DH \&WQS & {$[24]$} \\
& Heuristic & DH \& WQS & {$[25]$} \\
& IP \& heuristic & DHS & {$[26]$} \\
& Heuristic & SHS & {$[27]$} \\
& Heuristic & SHS & {$[15]$} \\
DC & IP & SHS & {$[17]$} \\
& Heuristic & SHS & {$[19]$} \\
& IP & DH \& WQS & {$[28]$} \\
& Heuristic & Not required & {$[29]$} \\
& Heuristic & SHS & {$[30]$} \\
& ACO & SHS & {$[31]$} \\
& Heuristic & SHS & {$[12],[15],[32]$} \\
PE & Branch \& bound & SHS & {$[33]$} \\
& Heuristic & DH \& WQS & {$[34]$} \\
& MIP & SHS & {$[21]$} \\
& L-shaped BONUS algorithm & DHS & {$[35]$} \\
& Stochastic decompostion & DHS & {$[36]$} \\
& GA & DH \& WQS & {$[37]$} \\
VC & Heuristic & DHS & {$[22]$} \\
& GA & DH \&WQS & {$[23]$} \\
& GA & DH \& WQS & {$[38]$} \\
\hline
\end{tabular}

IP: integer programming, ACO: ant colony optimisation, GA: generic algorithm, MIP: mixed integer programming, DHS: dynamic hydraulic simulation, EC: extent of contamination, TD: time to detection, DC: demand coverage, PE: population exposed, DHS \& WQS: dynamic hydraulic simulation and water quality simulation, SHS: static hydraulic simulation, AR: attack risk, WQS: water quality simulation, PD: probability density.

\subsection{Multi-objective}

The desire to reduce the number of sensors deployed on the network and also to protect the society from the use of contaminated water has continue to gain a significant recognition 
among the relevant stakeholders. Despite a series of enhancement made on the single objective strategies, a multi-objective approach is highly important. Accordingly, various multi-objectives strategies are briefly highlighted in this section. Berry et al. [39] and Propato [40] formulated a multi-objective Mixed Integer Programming (MIP) strategy for sensor placement in WDNs. Subsequently, Berry et al. [41] discussed how an undetected event by faulty sensors can be modelled and incorporated into a MIP formulation. Two notable approaches are widely considered in the formulation of multi-objectives approaches.

The first objective functions keeps the discrete and expressed results in term of the Pareto front [42]-[48]. The study by Dorini et al. [49] presented an efficient multi-objective algorithm for sensor placement in WDN. Four major objectives were considered in the model. These include: TD, VC, EC, and PE. The solution to the algorithm was based on a Noisy Cross-Entropy Sensor Locator (nCESL). The strategy was demonstrated on two water networks and excessive computational burden was identified as a concern. The study by Aral et al. [50] considered TD, VC, and maximising the detection likelihood in their formulation. The authors proposed a sub-domain concept in order to deal with the challenge of large network and to determine an accurate positioning. The techniques was based on the Non-dominated Sorting Generic Algorithm (NSGA-II) and two networks were used to examine the performance of the proposed algorithm. Preis and Ostfeld [44] developed multi-objective NSGA-II, based on the two objectives. The two objectives considered are: minimising the degree of consuming contaminated water following the detection and minimising the number of activities required to flush out the contaminant from the system. An application of the technique on two sample networks demonstrated its efficiency. However, the challenge of computational burden and the integration of uncertainty in the sensor were recommended by the authors. Similarly, a dual objective optimisation procedure was presented by Weickgenannt et al. [45]. The objectives were to minimise the number of sensors and to minimise the risk of contamination. The authors employed the NSGA-II in order to solve the problem and the water distribution network of Almelo in the Netherlands was examined as a case study.

The second approach deals with the integration of various objectives and treated as a single objective function by employing optimisation solver. Some authors [51]-[54] discussed such an approach. An efficient sensor placement which considered the timedetection and population exposed to contaminated water was presented by Krause et al. [51]. The study by Aral et al. [52] presented a simulation-optimisation design based on four performance objectives. They employed a progressive generic algorithm scheme in order to overcome the challenge of computational time requirement. A summary of some of multiobjective strategies is presented in Table 2.

\section{EXISTING SOLUTION APPROACHES}

The existing solution strategies are broadly split into four (4) major categories. These are briefly discussed in this section.

\subsection{Opinion-based}

This concept relies on the application of an expert opinion in order to make a decision on the sensor placement in water distribution networks. Such idea was employed in the study presented by Trachtman [63]. It however failed to meet standard when compared with optimisation-based strategy by Berry et al. [64]. An approach like this requires robust data which makes it less popular when compared to others that are popularly adopted. 
Table 2: Summary of some of the multi-objective approaches adapted from [12].

\begin{tabular}{llll}
\hline Objective & Approach & WQS & Reference \\
\hline TD, DL & NSGA-II \& GA & DH \& WQS & {$[55]$} \\
TD, DL & MOGA & DH \& WQS & {$[56]$} \\
TD, PE & Greedy and SA & DH \& WQS & {$[57]$} \\
TD, VC, PE, EC, and PFD & MIP and heuristic & DH \&WQS & {$[40]$} \\
TD, PE, VC, DL & Modified cross-entropy algorithm & DH \&WQS & {$[49]$} \\
TD, VC, PE, MC & GA & DH \&WQS & {$[58]$} \\
PE, MC & IP, local search \& NLP & DH \&WQS & {$[41]$} \\
TD, PE, VC, DL & MIP & DH \&WQS & {$[40]$} \\
TD, PE, DL & GA & DH \&WQS & {$[43]$} \\
TD, DL, SDR & NSGA-II & DH \&WQS & {$[59]$} \\
TD, DL, SDR & NSGA-II & DH \&WQS & {$[44]$} \\
TD, VC, DL & PGA & DH \&WQS & {$[50]$} \\
VC, NFD & NSGA-II & DH \&WQS & {$[46]$} \\
DL, PE & Heuristic & SH \&WQS & {$[60]$} \\
DL, PE & Heuristic & DH \&WQS & {$[53]$} \\
VC, NFD & NSGA-II & DH \&WQS & {$[45]$} \\
TD, VC, DL & GA & DH \&WQS & {$[61]$} \\
TD,DL, SDR, CSDL & NSGA-II & DH \&WQS & {$[62]$} \\
DL or CSDL & MIP & DH \&WQS & {$[53]$} \\
DC, TD, VC, PE, DL & Heuristic & DH \&WQS & {$[66]$} \\
Time delay, SDR & NSGA-II & DH \&WQS & {$[47]$} \\
\hline
\end{tabular}

NSGA: non-linear sorted generic algorithm, GA: generic algorithm, IP: integer programming, NLP: non-linear programming, MIP: mixed integer programming, PGA: progressive generic algorithm, SH: static hydraulic, $\mathrm{SH}$ \& WQS: static hydraulic and water quality simulation, WQS: water quality simulation, MOGA: multi-objective generic algorithm, PFD: probability of failed detection others acronyms are in the text.

\subsection{Theory-based}

The theory-based approach has only been utilised by few researchers in the sensor placement strategies. Christodoulou et al. [65] discussed an entropy-based theory in the sensor placement in WDNs. An application of diminishing marginal returns was proposed by Shen and McBean [67] in order to maximise the sensor detection redundancy due to wrong data.

\subsection{Rule-based}

In the rule-based strategies, the influence of suitable hydraulic and quality model can be negligible. This makes the method to be acceptable for a large distribution network. Some studies assessed the application of the rule-based approach [68], [69]. An application of a rule-based decision support system (RBDSS) was discussed by Chang et al. [70]. Reduction in computational time was an advantage of the approach when compared to other methods. However, the approach appears to be rather unrefined as it does not consider nodal demand. Thus, an optimisation-based strategy was suggested by the authors.

\subsection{Optimisation-based}

Optimisation-based approach has been widely adopted and can be categorised into: deterministic optimisation, stochastic optimisation, and robust optimisation. The studies by Kumar et al. [14] and Krause et al. [51] employed the deterministic algorithm for sensor layout. An excessive computational burden was outlined as a shortcoming of the method. Contrarily, a stochastic approach was discussed by few authors [35], [58], [71]. Although, some of the authors challenged the use of the method for worst scenario as it demands some presumed objective function. Thus, a robust optimisation method was suggested to handle 
the impediment. The work by Ma et al. [72] presented a mixed-integer programming method, which rely on the absolute robustness situation. Sensor placement strategy under various water demand criteria for robust quality monitoring was presented by Łangowski et al. [73]. For effective performance, an optimisation-based methods require integrating optimisation approach with hydraulic and water quality model, such as EPANET by Rossman et al. [74]. This will express the flow of water and the contaminants characteristic in WDN in order to ascertain where sensors can be installed.

\section{TECHNICAL CHALLENGES AND PROBABLE SOLUTIONS}

The present sensor placement strategies have some complications which must be addressed. This section outlined the challenges and proposed probable solutions.

\subsection{Excessive computational burden}

Majority of the aforementioned studies debated the computational burden as one of the key technical challenge when computing. Eliminating some nodes without compromising the sensor placement reliability is a viable alternative. Clustering of nodes and placement of sensor cluster-wise may be suitable [68] and thus, proposed.

\subsection{Choice of performance objectives}

The choice of a single performance objective may be insufficient. Thus, multi-objectives may be an alternative and subsequently increase the computational burden. Thus, a balance should be found between the competitive and complementary objectives. An adoption of such was discussed by Aral et al. [52] and hence suggested.

\subsection{Anomalies in sensor accuracy}

Even though factors of uncertainties have been considered by some authors [75], [76]. It is possible that sensor can give wrong signal due to susceptibility and corrosion [41]. Therefore, further investigation on the use of optimal choice of sensor (in terms of quality) is also recommended.

\section{FUTURE RESEARCH DIRECTIONS}

In spite of the advancement on the sensor placement strategies in WDNs, the research in this domain is still at an infancy stage. Hence, more effort is necessary. In this section, a summary of the further research directions are drawn.

\subsection{Hybrid formulation}

The study by Hamam and Brameller [77] had previously proposed a hybrid approach as a measure to address water piping systems. Thereafter, several optimisation techniques have been suggested to address the challenges of sensor placement in WDNs. Deterministic method was found reliable for sensor placement in small networks, but hitch in large network scenarios. In contrast, heuristic solvers can not guarantee the optimal solution, but can speedily resolve an optimal solution. Therefore, integration of the deterministic and heuristic in the form of a hybrid can be idea for optimisation in WDN. Also, combination of optimisation-based and rule-based methods is a promising alternative. Comparison of these methods is essential for a broad understanding of the strengths and weaknesses of each method in order to propose an enhance hybridised formulation method. 


\subsection{Consideration of parallel computing}

Excessive computation has been debated to be one of the major challenges of the sensor placement in a large water distribution network. However, the emergence of cloud computing has proved to be a resourceful alternative. The study by Tabaa et al. [78] embraced it and found it feasible. Therefore, sustenance of this technique is a way forward and hence, it is suggested.

\subsection{Utilisation of emerging technologies}

Some researchers have suggested the use of Artificial Neutral Networks (ANNs) and Software Defined Network (SDN) to handle some difficulties in WDNs [8], [79]-[81]. The implementation of such technology for water quality monitoring is yet to be fully explored. Therefore, more future improvement in this direction is strongly recommended.

\section{CONCLUSION}

The security of water distribution system has raised a level of concern after the terrorist attack in 2001. This has motivated researchers and relevant stakeholders in order to develop various methodologies that will improve the security of the water system. Enhancing the physical infrastructure and deployment of contaminant identification sensors on the water network are the two notable possibilities to curtain the insecurity. It is crucial to deploy water quality monitoring sensors across the water network. Nevertheless, complexity of the system, budget constraints, sensor placement issue and many others are essential to be addressed. As such, researchers have proposed various strategies for sensor placements considering the budget constraints. Despite this effort, there is no unified agreement amongst them on the various issues associated to the challenge. To this, the current study presents a critical review of the existing methodologies with the aim of outlining the technical challenges and proffer probable solutions. Generally speaking, advantages of the optimal sensor placement includes; minimization the cost of sensor procurement and significant reduction of the related effect of consuming contaminated water on the public health. Unfortunately, a thorough investigation through literature failed to establish readiness of Africa researchers to prevent probable water network attack as depicted in Tables 1 and 2. Besides the explicit outline of the existing scheme, the study discussed and summarized key technical challenges and present possible solutions. Based on an extensive understanding of the challenge, the study also provides area of further studies. Consequently, more effort should be committed to address the challenges related to sensor placement strategies and it is hereby recommended.

\section{ACKNOWLEDGEMENTS}

The authors will like to thank the Tshwane University of Technology, and the French South African Institute of Technology (F'SATI), Pretoria, South Africa for financial support.

\section{REFERENCES}

[1] Adedoja, O.S., Hamam, Y., Khalaf, B. \& Sadiku, R., Towards development of an optimization model to identify contamination source in a water distribution network. Water, 10(5), p. 579, 2018.

[2] Hrudey, S., Payment, P., Huck, P., Gillham, R. \& Hrudey, E., A fatal waterborne disease epidemic in Walkerton, Ontario: comparison with other waterborne outbreaks in the developed world. Water Science and Technology, 47(3), pp. 7-14, 2003.

[3] Cooper, W.J., Responding to crisis: the West Virginia chemical spill. ACS Publications, 2014. 
[4] Corso, P.S., Kramer, M.H., Blair, K.A., Addiss, D.G., Davis, J.P. \& Haddix, A.C., Costs of illness in the 1993 waterborne cryptosporidium outbreak, Milwaukee, Wisconsin. Emerging Infectious Diseases, 9(4), p. 426, 2003.

[5] Yazdi, J., Water quality monitoring network design for urban drainage systems, an entropy method. Urban Water Journal, 15(3), pp. 227-233, 2018.

[6] Adedeji, K.B., Hamam, Y., Abe, B.T. \& Abu-Mahfouz, A.M., Leakage detection and estimation algorithm for loss reduction in water piping networks. Water, 9(10), p. 773, 2017.

[7] Rathi, S. \& Gupta, R., Optimal sensor locations for contamination detection in pressuredeficient water distribution networks using genetic algorithm. Urban Water Journal, 14(2), pp. 160-172, 2017.

[8] Hamam, Y. \& Hindi, K., Optimised on-line leakage minimisation in water piping networks using neural nets. Proceedings of the IFIP Working Conference, Dagschul, Germany, vol. 28, pp. 57-64, 1992.

[9] Farley, B., Mounce, S. \& Boxall, J., Field testing of an optimal sensor placement methodology for event detection in an urban water distribution network. Urban Water Journal, 7(6), pp. 345-356, 2010.

[10] Hindi, K. \& Hamam, Y., Locating pressure control elements for leakage minimisation in water supply networks by genetic algorithms. Artificial Neural Nets and Genetic Algorithms, Springer, pp. 583-587, 1993.

[11] Murray, R., Haxton, T., Janke, R., Hart, W.E., Berry, J. \& Phillips, C., Sensor Network Design for Drinking Water Contamination Warning Systems: A Compendium of Research Results and Case Studies using the TEVA-SPOT Software. National Homeland Security Research Center, US Environmental Protection Agency, Cincinnati, OH, USA, 2010.

[12] Rathi, S. \& Gupta, R., Sensor placement methods for contamination detection in water distribution networks: A review. Procedia Engineering, 89, pp. 181-188, 2014.

[13] Hu, C., Li, M., Zeng, D. \& Guo, S., A survey on sensor placement for contamination detection in water distribution systems. Wireless Networks, 24(2), pp. 647-661, 2016.

[14] Kumar, A., Kansal, M., Arora, G., Ostfeld, A. \& Kessler, A., Detecting accidental contaminations in municipal water networks. Journal of Water Resources Planning and Management, 125(5), pp. 308-310, 1999.

[15] Rathi, S. \& Gupta, R., Monitoring stations in water distribution systems to detect contamination events. ISH Journal of Hydraulic Engineering, 20(2), pp. 142-150, 2014.

[16] Hu, C.Y., Tian, D.J., Liu, C. \& Yan, X., Sensors placement in water distribution systems based on co-evolutionary optimization algorithm. 1st International Conference on Industrial Networks and Intelligent Systems (INISCom), IEEE, pp. 7-11, 2015.

[17] Lee, B.H. \& Deininger, R.A., Optimal locations of monitoring stations in water distribution system. Journal of Environmental Engineering, 118(1), pp. 4-16, 1992.

[18] Liu, S., Liu, W., Chen, J. \& Wang, Q., Optimal locations of monitoring stations in water distribution systems under multiple demand patterns: a flaw of demand coverage method and modification. Frontiers of Environmental Science \& Engineering, 6(2), pp. 204-212, 2012.

[19] Kumar, A., Kansal, M. \& Arora, G., Identification of monitoring stations in water distribution system. Journal of Environmental Engineering, 123(8), pp. 746-752, 1997.

[20] Propato, M., Piller, O. \& Uber, J., A sensor location model to detect contaminations in water distribution networks. Impacts of Global Climate Change, pp. 1-12, 2005. 
[21] Berry, J.W., Fleischer, L., Hart, W.E., Phillips, C.A. \& Watson, J.P., Sensor placement in municipal water networks. Journal of Water Resources Planning and Management, 131(3), pp. 237-243, 2005.

[22] Kessler, A., Ostfeld, A. \& Sinai, G., Detecting accidental contaminations in municipal water networks. Journal of Water Resources Planning and Management, 124(4), pp. 192-198, 1998.

[23] Ostfeld, A. \& Salomons, E., Optimal layout of early warning detection stations for water distribution systems security. Journal of Water Resources Planning and Management, 130(5), pp. 377-385, 2004.

[24] Cozzolino, L., Mucherino, C., Pianese, D. \& Pirozzi, F., Positioning, within water distribution networks, of monitoring stations aiming at an early detection of intentional contamination. Civil Engineering and Environmental Systems, 23(3), pp. 161-174, 2006.

[25] Chastain Jr, J.R., Methodology for locating monitoring stations to detect contamination in potable water distribution systems. Journal of Infrastructure Systems, 12(4), pp. 252259, 2006.

[26] Pinzinger, R., Deuerlein, J., Wolters, A. \& Simpson, A., Alternative approaches for solving the sensor placement problem in large networks. World Environmental and Water Resources Congress 2011: Bearing Knowledge for Sustainability, pp. 314-323, 2011.

[27] Kansal, M., Dorji, T., Chandniha, S.K. \& Tyagi, A., Identification of optimal monitoring locations to detect accidental contaminations. World Environmental and Water Resources Congress 2012: Crossing Boundaries, pp. 758-776, 2012.

[28] Alhumaizi, K., Henda, R. \& Soliman, M., Numerical analysis of a reaction-diffusionconvection system. Computers \& Chemical Engineering, 27(4), pp. 579-594, 2003.

[29] Ghimire, S.R. \& Barkdoll, B.D., Heuristic method for the battle of the water network sensors: Demand based approach. Water Distribution Systems Analysis Symposium 2006, pp. 1-10, 2008.

[30] Kansal, M., Dorji, T. \& Chandniha, S.K., Design scheme for water quality monitoring in a distribution network. Int. J. Environ. Dev., 9(1), pp. 69-81, 2012.

[31] Afshar, A. \& Khombi, S.M., Multiobjective optimization of sensor placement in water distribution networks; dual use benefit approach. Int. J. Optim. Civil. Eng., 5(3), pp. 315-331, 2015.

[32] Rathi, S. \& Gupta, R., Locations of sampling stations for water quality monitoring in water distribution networks. Journal of Environmental Science \& Engineering, 56(2), pp. 169-178, 2014.

[33] Carr, R.D., Greenberg, H.J., Hart, W.E. \& Phillips, C.A., Addressing modeling uncertainties in sensor placement for community water systems. Critical Transitions in Water and Environmental Resources Management, pp. 1-10, 2004.

[34] Uber, J., Janke, R., Murray, R. \& Meyer, P., Greedy heuristic methods for locating water quality sensors in distribution systems. Critical Transitions in Water and Environmental Resources Management, pp. 1-9, 2004.

[35] Shastri, Y. \& Diwekar, U., Sensor placement in water networks: A stochastic programming approach. Journal of Water Resources Planning and Management, 132(3), pp. 192-203, 2006.

[36] Rico-Ramirez, V., Frausto-Hernandez, S., Diwekar, U.M. \& Hernandez-Castro, S., Water networks security: A two-stage mixed-integer stochastic program for sensor placement under uncertainty. Computers \& Chemical Engineering, 31(5), pp. 565-573, 2007. 
[37] Schwartz, R., Lahav, O. \& Ostfeld, A., Optimal sensor placement in water distribution systems for injection of chlorpyrifos. World Environmental and Water Resources Congress 2014, pp. 485-494, 2014.

[38] Ostfeld, A. \& Salomons, E., Optimal early warning monitoring system layout for water networks security: inclusion of sensors sensitivities and response delays. Civil Engineering and Environmental Systems, 22(3), pp. 151-169, 2005.

[39] Berry, J., Hart, W.E., Phillips, C.A., Uber, J.G. \& Watson, J.P., Sensor placement in municipal water networks with temporal integer programming models. Journal of Water Resources Planning and Management, 132(4), pp. 218-224, 2006.

[40] Propato, M., Contamination warning in water networks: General mixed-integer linear models for sensor location design. Journal of Water Resources Planning and Management, 132(4), pp. 225-233, 2006.

[41] Berry, J., Carr, R.D., Hart, W.E., Leung, V.J., Phillips, C.A. \& Watson, J.P., Designing contamination warning systems for municipal water networks using imperfect sensors. Journal of Water Resources Planning and Management, 135(4), pp. 253-263, 2009.

[42] Eliades, D. \& Polycarpou, M., Iterative deepening of Pareto solutions in water sensor networks. Water Distribution Systems Analysis Symposium 2006, pp. 1-19, 2008.

[43] Huang, J.J., McBean, E.A. \& James, W., Multi-objective optimization for monitoring sensor placement in water distribution systems. Water Distribution Systems Analysis Symposium 2006, pp. 1-14, 2008.

[44] Preis, A. \& Ostfeld, A., Multiobjective sensor design for water distribution systems security. Water Distribution Systems Analysis Symposium 2006, pp. 1-17, 2008.

[45] Weickgenannt, M., Kapelan, Z., Blokker, M. \& Savic, D.A., Risk-based sensor placement for contaminant detection in water distribution systems. Journal of Water Resources Planning and Management, 136(6), pp. 629-636, 2010.

[46] Guidorzi, M., Franchini, M. \& Alvisi, S., A multi-objective approach for detecting and responding to accidental and intentional contamination events in water distribution systems. Urban Water Journal, 6(2), pp. 115-135, 2009.

[47] Shen, H. \& McBean, E., Pareto optimality for sensor placements in a water distribution system. Journal of Water Resources Planning and Management, 137(3), pp. 243-248, 2010.

[48] Naserizade, S.S., Nikoo, M.R. \& Montaseri, H., A risk-based multi-objective model for optimal placement of sensors in water distribution system. Journal of Hydrology, 557, pp. 147-159, 2018.

[49] Dorini, G., Jonkergouw, P., Kapelan, Z., Di Pierro, F., Khu, S. \& Savic, D., An efficient algorithm for sensor placement in water distribution systems. Water Distribution Systems Analysis Symposium 2006, pp. 1-13, 2008.

[50] Aral, M.M., Guan, J. \& Maslia, M.L., A multi-objective optimization algorithm for sensor placement in water distribution systems. World Environmental and Water Resources Congress 2008: Ahupua'A, pp. 1-11, 2008.

[51] Krause, A., Leskovec, J., Guestrin, C., VanBriesen, J. \& Faloutsos, C., Efficient sensor placement optimization for securing large water distribution networks. Journal of Water Resources Planning and Management, 134(6), pp. 516-526, 2008.

[52] Aral, M.M., Guan, J. \& Maslia, M.L., Optimal design of sensor placement in water distribution networks. Journal of Water Resources Planning and Management, 136(1), pp. 5-18, 2009.

[53] Xu, J., Johnson, M.P., Fischbeck, P.S., Small, M.J. \& VanBriesen, J.M., Robust placement of sensors in dynamic water distribution systems. European Journal of Operational Research, 202(3), pp. 707-716, 2010. 
[54] Margarida, D. \& Antunes, C.H., Multi-objective optimization of sensor placement to detect contamination in water distribution networks. Proceedings of the Companion Publication of the 2015 Annual Conference on Genetic and Evolutionary Computation, ACM, pp. 1423-1424, 2015.

[55] Ehsani, N. \& Afshar, A., Application of NA-ACO in multiobjective contaminant sensor network design for water distribution systems. Water Distribution Systems Analysis 2010, pp. 327-337, 2010.

[56] Kim, J.H., Tran, T.V.T. \& Chung, G., Optimization of water quality sensor locations in water distribution systems considering imperfect mixing. Water Distribution Systems Analysis 2010, pp. 317-326, 2010.

[57] Krause, A. \& Guestrin, C., Robust sensor placement for detecting adversarial contaminations in water distribution systems. World Environmental and Water Resources Congress 2009: Great Rivers, pp. 1-10, 2009.

[58] Cozzolino, L., Della Morte, R., Palumbo, A. \& Pianese, D., Stochastic approaches for sensors placement against intentional contaminations in water distribution systems. Civil Engineering and Environmental Systems, 28(1), pp. 75-98, 2011.

[59] Austin, R.G., Choi, C.Y., Preis, A., Ostfeld, A. \& Lansey, K., Multi-objective sensor placements with improved water quality models in a network with multiple junctions. World Environmental and Water Resources Congress 2009: Great Rivers, pp. 1-9, 2009.

[60] Dorini, G., Jonkergouw, P., Kapelan, Z. \& Savic, D., Slots: Effective algorithm for sensor placement in water distribution systems. Journal of Water Resources Planning and Management, 136(6), pp. 620-628, 2010.

[61] Wu, Z. \& Walski, T., Multi objective optimization of sensor placement in water distribution systems. Water Distribution Systems Analysis Symposium, 2008.

[62] Preis, A. \& Ostfeld, A., Efficient contamination events sampling for sensors layout design. World Environmental and Water Resources Congress 2007: Restoring Our Natural Habitat, pp. 1-8, 2007.

[63] Trachtman, G.B., A "strawman" common sense approach for water quality sensor site selection. Water Distribution Systems Analysis Symposium 2006, pp. 1-13, 2008.

[64] Berry, J.W., Hart, W.E., Phillips, C.A., Uber, J.G. \& Walski, T.M., Water quality sensor placement in water networks with budget constraints. Impacts of Global Climate Change, pp. 1-11, 2005.

[65] Christodoulou, S.E., Gagatsis, A., Xanthos, S., Kranioti, S., Agathokleous, A. \& Fragiadakis, M., Entropy-based sensor placement optimization for waterloss detection in water distribution networks. Water Resources Management, 27(13), pp. 4443-4468, 2013.

[66] Eliades, D., Kyriakou, M. \& Polycarpou, M., Sensor placement in water distribution systems using the S-PLACE toolkit. Procedia Engineering, 70, pp. 602-611, 2014.

[67] Shen, H. \& McBean, E., Diminishing marginal returns for sensor networks in a water distribution system. Journal of Water Supply: Research and Technology-Aqua, 60(5), pp. 286-293, 2011.

[68] Perelman, L. \& Ostfeld, A., Water-distribution systems simplifications through clustering. Journal of Water Resources Planning and Management, 138(3), pp. 218229, 2011.

[69] Chang, N.B., Pongsanone, N.P. \& Ernest, A., A rule-based decision support system for sensor deployment in small drinking water networks. Journal of Cleaner Production, 29, pp. 28-37, 2012. 
[70] Chang, N.B., Prapinpongsanone, N. \& Ernest, A., Optimal sensor deployment in a largescale complex drinking water network: Comparisons between a rule-based decision support system and optimization models. Computers \& Chemical Engineering, 43, pp. 191-199, 2012.

[71] Comboul, M. \& Ghanem, R., Value of information in the design of resilient water distribution sensor networks. Journal of Water Resources Planning and Management, 139(4), pp. 449-455, 2012.

[72] Ma, X., Song, Y., Huang, J. \& Wu, J., Robust sensor placement problem in municipal water networks. Third International Joint Conference on Computational Science and Optimization (CSO), IEEE, vol. 1, pp. 291-294, 2010.

[73] Łangowski, R., Brdys, M.A. \& Qi, R., Optimised robust placement of hard quality sensors for robust monitoring of quality in drinking water distribution systems. 10th World Congress on Intelligent Control and Automation (WCICA), IEEE, pp. 1109-1114, 2012.

[74] Rossman, L.A. et al., Epanet 2: Users Manual. US Environmental Protection Agency, Office of Research and Development. National Risk Management Research Laboratory, 2000.

[75] Salomons, E. \& Ostfeld, A., Identification of possible contamination sources using reverse hydraulic simulation. Water Distribution Systems Analysis 2010, pp. 447-453, 2010.

[76] Mukherjee, R., Diwekar, U.M. \& Vaseashta, A., Optimal sensor placement with mitigation strategy for water network systems under uncertainty. Computers \& Chemical Engineering, 103, pp. 91-102, 2017.

[77] Hamam, Y. \& Brameller, A., Hybrid method for the solution of piping networks. Proceedings of the Institution of Electrical Engineers, 118(11), pp. 1607-1612, 1971.

[78] Tabaa, Y., Medouri, A. \& Tetouan, M., Towards a next generation of scientific computing in the cloud. International Journal of Computer Science, 9(6), pp. 177-183, 2012.

[79] Zhuiykov, S., Solid-state sensors monitoring parameters of water quality for the next generation of wireless sensor networks. Sensors and Actuators B: Chemical, 161(1), pp. 1-20, 2012.

[80] Lambrou, T.P., Anastasiou, C.C., Panayiotou, C.G. \& Polycarpou, M.M., A low-cost sensor network for real-time monitoring and contamination detection in drinking water distribution systems. IEEE Sensors Journal, 14(8), pp. 2765-2772, 2014.

[81] Perelman, L. \& Ostfeld, A., Operation of remote mobile sensors for security of drinking water distribution systems. Water Research, 47(13), pp. 4217-4226, 2013. 